\title{
Values of Monte Carlo Samples for Geotechnical Reliability-Based Design
}

\author{
Zi-Jun $\mathrm{Cao}^{1}$, Guo-Hui Gao ${ }^{1}$, Dian-Qing $\mathrm{Li}^{1}$, and $\mathrm{Yu} \mathrm{Wang}^{2}$
}

${ }^{1}$ State Key Laboratory of Water Resources and Hydropower Engineering Science, Institute of Engineering Risk and Disaster Prevention, Wuhan University, 8 Donghu South Road, Wuhan 430072, P. R. China.

E-mail: zijuncao@whu.edu.cn (corresponding author); guohui.gao@whu.edu.cn; dianqing@whu.edu.cn

${ }^{2}$ Department of Architecture and Civil Engineering, City University of Hong Kong, Tat Chee Avenue, Kowloon, Hong Kong E-mail: yuwang@,cityu.edu.cn

\begin{abstract}
With the advent of computing technologies, Monte Carlo simulation (MCS) has been gaining wide applications in geotechnical engineering. Various MCS techniques (e.g., direct MCS, Subset Simulation (SS), and Generalized SS (GSS)) have been successfully applied to developing full probabilistic design methodologies for geotechnical reliability-based design (RBD). Nevertheless, geotechnical practitioners are reluctant to adopt full probabilistic design methodologies due to conceptual and mathematical hurdles of reliability algorithms. This paper presents recent attempts aiming at improving the practical applicability of MCS-based full probabilistic RBD approaches by decoupling reliability algorithms from deterministic design calculations and implementing MCS-based reliability calculations in an EXCEL spreadsheet environment. Besides reliability calculations, post-processing MCS samples allows achieving efficient RBD updating under different design scenarios, providing reliability sensitivity of uncertain parameters to identify the most significant uncertainty sources, and determining design points of uncertain parameters from their failure samples for bridging full and semiprobabilistic RBD methodologies. More attentions shall be paid to these values of MCS samples in future to take advantage of fast development of high performance computing.
\end{abstract}

Keywords: Monte Carlo simulation; reliability-based design; design point; geotechnical structure; EXCEL spreadsheet.

\section{Introduction}

Reliability-based design (RBD) provides a coherent and systematic framework for incorporating various geotechnical-related uncertainties (e.g., uncertainties in geotechnical properties and loads, model uncertainty, etc.) into the design process. One key goal in geotechnical RBD for a given design problem is to achieve a uniform reliability level close to the target reliability under various design scenarios that are specified by uncertain soil parameters, load conditions, and limit state functions (ISO2394 2015). Nevertheless, geotechnical design scenarios can vary over a wide range due to site-specific nature and relatively large variability of geotechnical materials, various design calculation models for the same design problem, and unknown failure modes among multiple correlated ones prior to design calculations (e.g., Phoon et al. 2016). These factors pose profound challenges in developing standardized semi-probabilistic RBD methodologies/codes for geotechnical structures, such as load and resistance factor design method and partial factor design method.

On the other hand, the above challenges encountered in semi-probabilistic RBD can be tackled with relative ease using full probabilistic RBD approaches. Under a full probabilistic design framework, reliability analysis methods (e.g., first order second moment (FOSM) method, first order reliability method (FORM), and Monte Carlo simulation (MCS) methods) are directly applied to calculating the probability of failure $P\left(F \mid D^{(j)}\right)$ (or reliability index $\beta^{(j)}$ ) of possible designs $D^{(j)}, j=1,2, \ldots, N_{D}$, in a design domain prescribed by geotechnical practitioners, and feasible designs are identified as those satisfying the target reliability requirement specified by the target probability of failure $P_{T}$ (or target reliability index $\beta_{T}$ ) (Gao et al. 2019). Although the full probabilistic RBD approach is considered particularly suitable for geotechnical designs, geotechnical practitioners are reluctant to adopt it in practice, at least partially, because of their limited training in probability and statistics and the hurdle of reliability algorithms which are often mathematically and computationally sophisticated (e.g., Phoon et al. 2016). This necessitates a practical and conceptually simple framework that allows extending conventional deterministic design calculations to full probabilistic RBD in a straightforward manner.

MCS provides a conceptually simple and robust method for solving geotechnical reliability problems in general. It can be simply viewed as a numerical process of repeatedly calculating a mathematical or empirical operator (e.g., the deterministic model needed in design) using random samples of uncertain parameters $\underline{\boldsymbol{X}}$, which are modeled as random variables with prescribed probability distributions, as input (Ang and Tang 2007). From the perspective of MCS, full probabilistic RBD process can be divided into to three parts: uncertainty modeling, deterministic modeling, and uncertainty propagation (i.e., reliability calculations) (Wang 2011; Wang and Cao 2013, 2015; Cao et al 2019; Gao et al. 2019). By this means, the uncertainty modeling and propagation are deliberately decoupled from the conventional deterministic design calculations so that they can proceed as an extension of the deterministic design calculations. This allows the deterministic model and uncertainty model to

\author{
Proceedings of the 7th International Symposium on Geotechnical Safety and Risk (ISGSR) \\ Editors: Jianye Ching, Dian-Qing Li and Jie Zhang \\ Copyright (c) ISGSR 2019 Editors. All rights reserved. \\ Published by Research Publishing, Singapore. \\ ISBN: 978-981-11-2725-0; doi:10.3850/978-981-11-2725-0_bs 1-cd
}


be developed separately by personnel with different expertise and in a parallel manner. Although this idea seems to be straightforward, MCS-based full probabilistic RBD approaches are often criticized because of (1) a lack of computational efficiency and resolution at small probability levels, such as feasible design domains with $P\left(F \mid D^{(j)}\right)$ less than $P_{T}$; (2) providing no insight into the relative contributions of various uncertainties to reliability calculations; (3) the difficulty in implementing MCS-based full probabilistic design approach in practice.

This paper presents recent attempts to tackle the above difficulties in MCS-based full probabilistic design so as to facilitate its practical implementation. One significant feature of MCS in comparison with other analytical and/or approximate reliability methods (e.g., FOSM and FORM) lies in random samples generated from probability density functions (PDFs) of uncertain parameters. Random samples generated by MCS not only numerically represent the PDFs of uncertain parameters (no matter how complex the PDFs are) but also allows performing complicated probabilistic reasoning in a convenient and discrete manner. This is an important value of MCS samples in the sense that it allows decoupling uncertainty modeling and propagation from deterministic design calculations for developing practical full probabilistic design tools based on MCS. MCS samples also provide more values beyond reliability calculations, such as efficient RBD updating under different design scenarios, reliability sensitivity information of uncertain parameters, design points for bridging full and semiprobabilistic RBD approaches, as discussed below.

\section{Full Probabilistic RBD Using Monte Carlo Simulation Methods}

RBD is referred to as "Design procedure that is subjected to prescribed reliability level of the structure" by ISO 2394: 2015 (E), Clause 2.1.29 (ISO2394, 2015). By this definition, the core task of MCS-based full probabilistic RBD is to determine feasible designs with $P\left(F \mid D^{(j)}\right)<P_{T}$, or equivalently $\beta^{(j)}>\beta_{T}$, among possible designs in a design domain prescribed by users. Consider, for example, a number, $N_{D}$, of possible designs $D^{(j)}, j=1,2, \ldots, N_{D}$, with dimensions rounded to some decimal for construction convenience (e.g., accommodation to dimensions of construction equipment). Each possible design $D^{(j)}$ is specified by a combination of design parameters, and may have a number, $N_{F}$, of failure modes $F_{n}^{(j)}, n=1,2, \ldots, N_{F}$, such as sliding along the base of a earth retaining wall, overturning about the toe of the wall, and bearing capacity failure of soils beneath the wall. Let $F^{(j)}$ denote the failure event of $D^{(j)}$ concerned in design, which can be either the failure event of geotechnical engineering systems or failure events corresponding to component failure modes of geotechnical structures, and its corresponding occurrence probability is represented by $P\left(F \mid D^{(j)}\right)$. Determining feasible designs with $P\left(F \mid D^{(j)}\right)<$ $P_{T}$ needs to calculate $P\left(F \mid D^{(j)}\right)$ values of possible designs. A straightforward, but computationally expensive, way to calculate $P\left(F \mid D^{(j)}\right)$ values of all possible designs in the design domain is to repeatedly perform $N_{D}$ runs of MCS, each of which re-generate MCS samples and re-evaluate design calculation models for each sample. Alternatively, $P\left(F \mid D^{(j)}\right)$ values of all possible designs can be obtained by post-processing MCS samples generated by a single MCS run so that repeatedly running MCS for different possible designs is avoided.

\subsection{Direct MCS for geotechnical RBD}

Direct MCS is the simplest MCS technique, and it is widely applied to probabilistic analyses and RBD of geotechnical structures due to its simplicity and robustness. Based on direct MCS, an expanded RBD framework (Wang 2011; Wang et al. 2011; Wang and Cao 2015; Cao et al. 2019) was proposed for full probabilistic design. It can be viewed as an augmented reliability analysis of a geotechnical structure, in which design parameters are artificially considered as uncertain with probability distributions specified by the user for design exploration purposes. For example, the design parameters $\underline{\boldsymbol{d}}$ can be represented as discrete uniform random variables. Each possible design $D^{(j)}$ is specified by a possible combination of discrete values of design parameters and has a probability of $P\left(D^{(j)}\right)$ given the prescribed probability distributions of design parameters. Then, the RBD process is formulated as a process of calculating $P\left(F \mid D^{(j)}\right)$ values of all the possible designs and comparing them with $P_{T}$. This can be achieved by a single run of direct MCS with the aid of Bayesian analysis of MCS samples, in which $P\left(F \mid D^{(j)}\right)$ is written as:

$$
P\left(F \mid D^{(j)}\right)=P\left(D^{(j)} \mid F\right) P(F) / P\left(D^{(j)}\right)
$$

where $P\left(D^{(j)} \mid F\right)=$ the occurrence probability of $D^{(j)}$ given failure; $P(F)=$ the failure probability considering failure samples of all possible designs. Since design parameters are represented as discrete uniform random variables, $P\left(D^{(j)}\right)$ is taken as equal to $1 / N_{D}$, where $N_{D}$ is the number of possible designs. During Direct MCS, random samples of both design parameters $\underline{\boldsymbol{d}}$ and uncertain parameters $\underline{\boldsymbol{X}}$ needed in design calculation models are simulated for expanded RBD. Then, failure samples and those samples corresponding to each $D^{(j)}$ are identified from random samples generated by a single run of direct MCS to estimate $P(F)$ and $P\left(D^{(j)} \mid F\right)$ of all the $N_{D}$ possible designs, respectively. To ensure the accuracy and resolution of estimated $P(F), P\left(D^{(j)} \mid F\right)$, and $P\left(F \mid D^{(j)}\right)$ in Eq. (1), a large number of direct MCS samples are often needed, and the number of random samples needed increases as the number of possible designs and the target reliability level increase. 


\subsection{Subset simulation for geotechnical RBD}

To improve the computational efficiency, the expanded RBD framework can be combined with advanced MCS techniques, such as Subset simulation (SS) (Au and Beck 2001; Wang and Cao 2013, 2015; Au and Wang 2014). SS converts a small failure probability into a product of a sequence of relativity large conditional probabilities by introducing intermediate events adaptively, and employs specially designed Markov chains to generate conditional samples of these intermediate events until the target failure domain is achieved (Au et al. 2010; Wang and Cao 2013; Li et al. 2016). SS starts with direct MCS and proceeds level by level. It divides sample space $\Omega$ of uncertain parameters (including $\underline{\boldsymbol{d}}$ and $\underline{\boldsymbol{X}}$ ) in expanded RBD into $m+1$ individual subsets $\left\{\Omega_{i}, i=0,1\right.$, $2, \ldots, m\}$ by the intermediate threshold values $\left\{y_{i}, i=1,2, \ldots, m\right\}$ of the driving variable $Y$, which is a key factor affecting the generation of conditional samples of interest in SS (Au and Wang 2014; Li et al. 2016). In SS, the $\left\{y_{\mathrm{i}}, \mathrm{i}=1,2, \ldots, m\right\}$ are adaptively determined, and samples in different subsets $\left\{\Omega_{i}, i=0,1,2, \ldots, m\right\}$ are generated level by level and correspond to different probability weights, i.e., $P\left(\Omega_{0}\right)=1-p_{0} ; P\left(\Omega_{i}\right)=p_{0}{ }^{i}-p_{0}{ }^{i+1}$ for $i=1, \ldots m-1$; and $P\left(\Omega_{m}\right)=p_{0}{ }^{m}$, where $p_{0}$ is a conditional probability (e.g., 0.1$)$. Hence, the $P(F)$ is written as:

$P(F)=\sum_{i=0}^{m} P\left(F \mid \Omega_{i}\right) P\left(\Omega_{i}\right)$

where $P\left(F \mid \Omega_{i}\right)=$ the conditional failure probability given sampling in $\Omega_{i} ; P\left(F \mid \Omega_{i}\right)$ is estimated as the ratio of the failure sample number in $\Omega_{i}$ over the total sample number in $\Omega_{i}$. In addition, using the Theorem of Total Probability, the conditional probability $P\left(D^{(j)} \mid F\right)$ in Eq. (1) is given by:

$P\left(D^{(j)} \mid F\right)=\sum_{i=0}^{m} P\left(D^{(j)} \mid F \cap \Omega_{i}\right) P\left(\Omega_{i} \mid F\right)$

where $P\left(\Omega_{i} \mid F\right)=$ the probability of failure samples falling into $\Omega_{i}$; and $P\left(D^{(j)} \mid F \cap \Omega_{i}\right)=$ the conditional probability of $D^{(j)}$ given sampling in $\Omega_{i}$ and the occurrence of failure, and it is expressed as the ratio of the number, $n_{f, i}^{(j)}$, of failure samples for $D^{(j)}$ in $\Omega_{i}$ over the total failure sample number $n_{f, i}$ in $\Omega_{i}$, i.e., $P\left(D^{(j)} \mid F \cap \Omega_{\mathrm{i}}\right)=n_{f, i}^{(j)} / n_{f, i}$. Using Eqs. (2) and (3), $P(F)$ and $P\left(D^{(j)} \mid F\right)$ are calculated using samples simulated from SS. Subsequently, $P\left(F \mid D^{(j)}\right)$ is obtained in accordance with Eq. (1). Compared with the direct MCS, SS efficiently generates a large number of failure samples to calculate $P(F)$ and $P\left(D^{(j)} \mid F\right)$. The SS-based expanded RBD requires to construct a design response $Y$ that simultaneously drives the sampling space to failure domains of all the possible designs. Constructing a robust design response $Y$ for SS-based expanded RBD might not be a trivial task, particularly when multiple correlated failure modes are involved in the design. Heuristic methods for constructing $Y$ in expanded RBD have been proposed in the literature (Wang and Cao 2013; Li et al. 2016).

\subsection{Generalized subset simulation for geotechnical RBD}

SS was recently extended to efficiently estimate the failure probabilities of multiple failure events by a single simulation run (Li et al. 2015; Gao et al. 2019), which is referred to as generalized Subset simulation (GSS). In the context of GSS, each possible design $D^{(j)}$ in the design space can be viewed as an equivalent failure event for RBD. The failure probabilities of all possible designs in design space can be calculated by a single GSS run without needs of Bayesian analysis given by Eq. (1) and deliberate choice of $Y$. For this purpose, GSS defines a unified failure event $F_{U}$, i.e., the union of failure events of the $N_{D}$ possible designs, i.e., $F_{U}=F^{(1)} \cup F^{(2)} \cup \ldots \cup F^{(j)} \cup \ldots \cup F^{\left(N_{D}\right)}$. Then, GSS turns to explore the unified failure domain (i.e., $F_{U}$ ) so as to simultaneously generate failure samples of different possible designs, details of which are referred to Gao et al. (2019). By this means, the failure domains (i.e., $\left.F^{(j)}, j=1,2, \ldots, N_{D}\right)$ of possible designs with different $P\left(F \mid D^{(j)}\right)$ values are reached at different simulation levels. Let $m^{(j)}$ denote the number of simulation levels needed to reach the failure domain $F^{(j)}$ of $D^{(j)}$. In general, $m^{(j)}$ increases with the decrease of $P\left(F \mid D^{(j)}\right)$. Using the samples generated by GSS, the $P\left(F \mid D^{(j)}\right)$ is estimated as:

$P\left(F \mid D^{(j)}\right)=P\left(F_{U, 1}\right) P\left(F_{U, 2} \mid F_{U, 1}\right) \cdots P\left(F_{U, m^{(j)}-1} \mid F_{U, m^{(j)}-2}\right) P\left(F^{(j)} \mid F_{U, m^{(j)}-1}\right)=\prod_{i=1}^{m^{(j)}-1} \frac{N_{i}}{N} \times \frac{N^{(j)}}{N}$

where $F_{U, i}=F_{i}^{(1)} \cup F_{i}^{(2)} \cup \ldots \cup F_{i}^{(j)} \cup \ldots \cup F_{i}^{\left(N_{D}\right)}, i=1,2, \ldots, m^{(j)}$, is the union of intermediate failure events (i.e., $F_{i}^{(j)}$, $\left.j=1,2, \ldots, N_{D}\right)$ of the $N_{D}$ designs in the $i$-th simulation level; $P\left(F_{U, 1}\right)=N_{1} / N$, and $N_{1}$ denotes the number of samples belong to $F_{U, 1}$ among the total number $N$ of samples generated in each simulation level during GSS; $P\left(F_{U, i} \mid F_{U, i-1}\right), i=2,3 \ldots, m^{(j)}-1$, is conditional probability of $F_{U, i}$ given sampling in $F_{U, i-1}$, and it is calculated as the ratio of the number $N_{i}$ of "seed" samples selected for the $i$-th level of Markov chain MCS among conditional samples in $F_{U, i-1}$ over $N$, i.e., $N_{i} / N ; P\left(F^{(j)} \mid F_{U, m^{(j-1}-1}\right)$ is conditional probability of $F^{(j)}$ given sampling in the unified intermediate event $F_{U, m^{(j)}-1}$, and is estimated as the ratio of the number $N^{(j)}$ of failure samples belonging to $F^{(j)}$ among conditional samples generated in $F_{U, m^{(j)}-1}$ over $N$. As $i$ increases, the failure domains of possible designs with relatively large $P\left(F \mid D^{(j)}\right)$ values are first arrived. After the failure domain of a particular design is reached, 
its intermediate failure event can be dropped from the unified intermediate failure event in subsequent simulation levels. Therefore, the number of possible designs considered in construction of $F_{U, i}$ decreases as $i$ increases. This leads to computational savings by avoiding calculating system responses of designs that have already been identified as failure.

GSS progressively drives the conditional sampling space to smaller probability levels with the increase of the number of simulation levels, and $P\left(F \mid D^{(j)}\right)$ values of possible designs obtained in each simulation level decrease. As the $P\left(F \mid D^{(j)}\right)$ values of possible designs obtained in a simulation level are less than $P_{T}$, the simulation can be terminated because the $P\left(F \mid D^{(j)}\right)$ values of remaining designs automatically satisfy the reliability requirement, i.e., less than $P_{T}$. By this means, feasible designs with $P\left(F \mid D^{(j)}\right)<P_{T}$ can be determined without explicitly calculating their $P\left(F \mid D^{(j)}\right)$ values. This saves considerable computational efforts since feasible designs have relatively small probabilities (less than $P_{T}$ ), calculations of which need more simulation levels. After feasible designs are determined, the optimal design is selected from them according to the design optimization criterion (e.g., minimization of construction costs) prescribed by geotechnical practitioners.

\section{Values of Monte Carlo Samples beyond Reliability Calculations}

MCS techniques provide random samples for numerical representation of the PDFs of uncertain parameters involved in RBD. These samples not only can be used to calculate the failure probabilities of different possible designs, but also provide more values for RBD updating under different design scenarios specified by different probability distributions and statistics of uncertain parameters, reliability sensitivity analysis of uncertain parameters, and determining their design points for a given limit state function. This section demonstrates how to obtain these values by post-processing Monte Carlo samples.

\subsection{RBD updating under different design scenarios}

The previous section presented full probabilistic RBD methodologies using MCS for a given design scenario. It is not uncommon to encounter a diversity of design scenarios for a given type of geotechnical structures due to various load conditions and the wide range of variability in geotechnical parameters. Let $f(\underline{\boldsymbol{X}})^{\mathrm{O}}$ and $f(\underline{\boldsymbol{X}})^{\mathrm{U}}$ denote the joint PDFs of uncertain parameters in the original and updated design scenarios, respectively. Consider, for example, that direct MCS-based expanded RBD (see Subsection 2.1) has been performed for full probabilistic design under the original design scenario specified by $f(\underline{\boldsymbol{X}})^{O}$, providing a number, $N_{T}^{(j)}$ of direct MCS samples of $\underline{\boldsymbol{X}}$ for $D^{(j)}$. As the design scenario changes, the failure probability of $D^{(j)}$ shall be updated as $P\left(F \mid D^{(j)}\right)^{U}$, which is written as:

$$
P\left(F \mid D^{(j)}\right)^{U}=\int I\left(F \mid D^{(j)}, \underline{\boldsymbol{X}}\right) f(\underline{\boldsymbol{X}})^{U} d \underline{\boldsymbol{X}}
$$

where $I\left(F \mid D^{(j)}, \underline{\boldsymbol{X}}\right)$ is the indicator function of the failure event of $D^{(j)}$ given $\underline{X} . I\left(F \mid D^{(j)}, \underline{X}\right)$ is equal to 1 if the failure of $D^{(j)}$ occurs; and otherwise, $I\left(F \mid D^{(j)}, \underline{\boldsymbol{X}}\right)$ is equal to zero. Using Eq. $(5), P\left(F \mid D^{(j)}\right)^{U}$ can be calculated by simulating random samples from $f(\underline{\boldsymbol{X}})^{U}$ by MCS. This, however, necessitates additional computational efforts for evaluating $I\left(F \mid D^{(j)}, \underline{\boldsymbol{X}}\right)$ for each sample and renders RBD updating a nontrivial task, particularly when a series of design scenarios are of interest in design. Such computational difficulties can be avoided by a sample reweighting technique, which allows using the random samples obtained from $f(\underline{\boldsymbol{X}})^{O}$ to calculate the $P\left(F \mid D^{(j)}\right)^{U}$ after the design scenario changes. In the context of the sample re-weighting technique, Eq. (5) is re-written as (Cao et al. 2019):

$$
P\left(F \mid D^{(j)}\right)^{U}=\int I\left(F \mid D^{(j)}, \underline{\boldsymbol{X}}\right) \frac{f(\underline{\boldsymbol{X}})^{U}}{f(\underline{\boldsymbol{X}})^{O}} f(\underline{\boldsymbol{X}})^{O} d \underline{\boldsymbol{X}} \approx \frac{1}{N_{T}^{(j)}} \sum_{i=1}^{N_{T}^{(j)}} I\left(F \mid D^{(j)}, \underline{\boldsymbol{X}}_{i}\right) \frac{f\left(\underline{\boldsymbol{X}}_{i}\right)^{U}}{f\left(\underline{\boldsymbol{X}}_{i}\right)^{O}}
$$

where $\underline{\boldsymbol{X}}_{i}, i=1,2, \ldots, N_{T}^{(j)}$ are the $N_{T}^{(j)}$ random samples of $\underline{\boldsymbol{X}}$ generated from $f(\underline{\boldsymbol{X}})^{O}$ (instead of $\left.f(\underline{\boldsymbol{X}})^{U}\right)$, and their corresponding values of $I\left(F \mid D^{(j)}, \underline{X}_{i}\right)$ have been evaluated before changing the design scenario. Based on Eq. (6), the updated failure probabilities of $D^{(j)}$ under different design scenarios specified by $f(\underline{\boldsymbol{X}})^{U}$ are calculated using the same set of random samples simulated for the original design scenario, avoiding re-sampling of $\underline{\boldsymbol{X}}$ from $f(\underline{\boldsymbol{X}})^{U}$ and re-evaluations of $I\left(F \mid D^{(j)}, \underline{\boldsymbol{X}}\right)$ for reliability updating under different design scenarios. By this means, the computational efficiency for MCS-based RBD updating can be improved substantially. This opens up a possibility of linking site investigation efforts with RBD updating using direct MCS in a cost-effective manner (e.g., Ching and Phoon 2012; Cao et al. 2019). It is worthwhile to point out that the sample re-weighting technique given by Eq. (6) shares the same mathematical formulation as the importance sampling. Their relationship and differences were discussed by Li et al. (2015) and are not pursued herein due to the limitation of paper length. 


\subsection{Reliability sensitivity of uncertain parameters}

Reliability sensitivity analysis aims to obtain insights into effects of uncertain parameters involved in design calculation models on reliability analysis results (e.g., probability of failure) of the geotechnical structure concerned. MCS was often criticized because it does not provide reliability sensitivity information of uncertain parameters (Baecher and Christian 2003). This section presents a probabilistic failure analysis approach that allows obtaining the reliability sensitivity information (i.e., effects of various uncertainties on failure probability) of uncertain parameters from failure samples generated by MCS. The probabilistic failure analysis approach contains two major components: hypothesis tests for prioritizing effects of various uncertain parameters and Bayesian analysis for further quantifying their effects, which are described below.

For a given design $D^{(j)}$, the probabilistic failure analysis approach prioritizes the effects of various uncertain parameters $\underline{\boldsymbol{X}}$ on the failure probability by comparing, statistically, failure samples with the $N_{T}^{(j)}$ unconditional samples of $D^{(j)}$ simulated by MCS. When the distribution of failure samples of an uncertain parameter deviates significantly from that of unconditional samples, the uncertainty in the parameter has a significant effect on $P\left(F \mid D^{(j)}\right)$. The deviation between the distribution of failure samples and that of unconditional samples can be quantified as below (Wang et al. 2010; Wang and Cao 2015):

$Z_{H, k}^{(j)}=\frac{\mu_{k}-\mu_{F, k}^{(j)}}{\sigma_{k} \sqrt{N_{F}^{(j)}}}$

where $\mu_{k}$ and $\sigma_{k}$ are the mean value and standard deviation of the uncertain parameter $X_{k} ; N_{F}^{(j)}$ is the number of failure samples for $D^{(j)}$ among a total of $N_{T}^{(j)}$ unconditional samples; and $\mu_{F, k}^{(j)}$ is the mean value of the $N_{F}^{(j)}$ failure samples of $X_{k}$ for $D^{(j)}$. When $\mu_{F, k}^{(j)}$ deviates significantly from $\mu_{k}$, the absolute value of $Z_{H, k}^{(j)}$ is relatively large. As the absolute value of $Z_{H, k}^{(j)}$ increases, the statistical difference between $\mu_{F, k}^{(j)}$ and $\mu_{k}$ becomes growingly significant. The effect of the uncertain parameter on failure probability also becomes growingly significant. The absolute value of $Z_{H, k}^{(j)}$ can therefore be used as an index to prioritize their relative effects on failure probability.

By comparing the absolute values of $Z_{H, k}^{(j)}$ for various uncertain parameters, the important uncertain parameters that have significant effects on failure probability are identified. Such effects can be explicitly quantified through the subsequent Bayesian analysis:

$P\left(F \mid X_{k}, D^{(j)}\right)=\frac{p\left(X_{k} \mid F, D^{(j)}\right) P\left(F \mid D^{(j)}\right)}{p\left(X_{k} \mid D^{(j)}\right)}$

where $P\left(F \mid X_{k}, D^{(j)}\right)$ is the conditional failure probability of $D^{(j)}$ for a given $X_{k}$ value; $p\left(X_{k} \mid F, D^{(j)}\right)$ is the conditional PDF of $X_{k}$ given that the failure of $D^{(j)}$ occurs; and $p\left(X_{k} \mid D^{(j)}\right)$ is the PDF of $X_{k}$ and is equal to $p\left(X_{k}\right)$ since $X_{k}$ is independent of $D^{(j)}$, which means that the PDF of $X_{k}$ remains the same for different possible designs. Eq. (8) implies that comparison between the $p\left(X_{k} \mid F, D^{(j)}\right)$ and $p\left(X_{k} \mid D^{(j)}\right)\left(\right.$ or $\left.p\left(X_{k}\right)\right)$ provides an indication of the effect of the uncertain parameter $X_{k}$ on the failure probability of $D^{(j)}$. Generally speaking, $P\left(F \mid X_{k}, D^{(j)}\right)$ changes as the value of the uncertain parameter $X_{k}$ changes. However, when $p\left(X_{k} \mid F, D^{(j)}\right)$ is similar to $p\left(X_{k}\right), P\left(F \mid X_{k}, D^{(j)}\right)$ remains more or less constant regardless of the values of $X_{k}$. This implies that the effect of $X_{k}$ on the failure probability of $D^{(j)}$ is minimal. Both hypothesis testing and Bayesian analysis results provide information on reliability sensitivity of uncertain parameters for a given design $D^{(j)}$.

\subsection{Identification of the design point among failure samples}

Design point plays a key role in reliability calculations and calibration of semi-probabilistic design codes (e.g., ISO2394 2015; Low and Phoon 2015). For a given design $D^{(j)}$, the design point of each component mode $F_{n}^{(j)}$ can be approximately determined from the failure samples according to its probabilistic interpretation, i.e., the most probable failure point (e.g., Shinozuka 1983; Low and Tang 1997; Der Kiureghian and Dakessian 1998). Depending on the joint PDF of uncertain parameters, the design point of $F_{n}^{(j)}$ can be determined in different ways. If the joint PDF of uncertain parameters is a multivariate normal distribution, the design point can be directly taken as the failure sample with the maximum joint PDF value (Shinozuka 1983). If uncertain parameters are non-normal, the design point of $F_{n}^{(j)}$ can be determined by minimizing the ellipsoid that touches the failure domain of $F_{n}^{(j)}$ in the original space of uncertain parameters according to the expanding ellipsoid perspective of FORM (e.g., Low and Tang 1997), which is achieved by minimizing the square root of the following quadratic form within the failure domain of $F_{n}^{(j)}$ : 
$\beta_{i}=\sqrt{\left[\left(\underline{\boldsymbol{X}}_{i}^{F}-\underline{\mu}_{i}^{N}\right) / \underline{\sigma}_{i}^{N}\right]^{T} R^{-1}\left[\left(\underline{\boldsymbol{X}}_{i}^{F}-\underline{\mu}_{i}^{N}\right) / \underline{\sigma}_{i}^{N}\right]}$

where $\underline{\boldsymbol{X}}_{i}^{F}$ is the $i$-th failure sample of $\underline{\boldsymbol{X}}$ for the failure mode $F_{n}^{(j)} ; R$ is the correlation matrix of $\underline{\boldsymbol{X}} ; \underline{\mu}_{i}^{N}$ and $\underline{\sigma}_{i}^{N}$ are, respectively, the equivalent normal mean value and standard deviation of $\underline{\boldsymbol{X}}$, each component of which corresponds to one uncertain parameter (e.g., $X_{k}$ ) and can be calculated by Rackwitz-Fiessler transformation (1978):

$\sigma_{k, i}^{N}=\phi\left\{\Phi^{-1}\left[G\left(X_{k, i}^{F}\right)\right]\right\} / f\left(X_{k, i}^{F}\right)$

$\mu_{k, i}^{N}=X_{k, i}^{F}-\sigma_{k, i}^{N} \Phi^{-1}\left[G\left(X_{k, i}^{F}\right)\right]$

where $\phi(\cdot)$ and $\Phi^{-1}[\cdot]$ are the PDF and inverse cumulative distribution function (CDF) of standard normal variable, respectively; $f\left(X_{k, i}^{F}\right)$ and $G\left(X_{k, i}^{F}\right)$ are the original PDF and CDF values evaluated at the $X_{k}$ value (i.e., $X_{k, i}^{F}$ ) of $\underline{\boldsymbol{X}}_{i}^{F}$, respectively. For the given $F_{n}^{(j)}$, the $\beta_{i}$ values of its corresponding failure samples $\underline{\boldsymbol{X}}_{i}^{F}$ generated by MCS are calculated using Eqs. (9)-(11). Then, the failure sample with the minimum $\beta_{i}$ value is approximately taken as the design point of non-normal uncertain parameters.

Note that the accuracy of the design point determined from the failure samples relies on the number of failure samples obtained during MCS. It generally improves as the number of failure samples increases if MCS is used to populate failure samples for numerical representation of the failure domain of the event of interest. In comparison with direct MCS, SS and GSS can efficiently generate a large number of failure samples with relative ease. More importantly, SS and GSS explore the sample space from the safe domain to failure domain in a progressive manner. This guarantees generating failure samples close to the limit state surface, where the most probable failure point often occurs if the joint PDF decays radially from the mean values of uncertain parameters.

\section{Implementation of MCS-Based Full Probabilistic Design in EXCEL Spreadsheet}

To facilitate the design practice, an EXCEL add-in, called GeoRBD/S, was developed to perform the MCSbased full probabilistic design. As shown in Figure 1, the GeoRBD/S includes not only a graphical user interface (GUI) to perform MCS but also worksheets for developing uncertainty and deterministic models deemed appropriate. Using GeoRBD/S, the implementation of MCS-based full probabilistic design is divided into three parts: uncertainty modeling, deterministic modeling, and uncertainty propagation, which are, respectively, achieved by uncertainty model worksheet, deterministic model worksheet, and MCS add-in.

As shown in Figure 1(a), the uncertainty model worksheet consists of three parts: (1) Zone 1 for defining random variables and design parameters; (2) Zone 2 for generating samples of random variables based on their statistical information in Zone 1; (3) Zone 3 for recording possible designs that are specified by combinations of possible values of design parameters provided in Zone 1. From the input-output (I-O) perspective, the uncertainty model worksheet takes statistical information (e.g., distribution types and statistics) of uncertain parameters and discrete values of design parameters as input, and returns a set of random samples of uncertain parameters and possible designs in design domain as output.

The deterministic model worksheet is developed for calculating system responses (e.g., $Y$ ) of geotechnical structures for a given set of system parameters, including, but not limited to, design parameters, design loads, and soil properties. As shown in Figure 1(c), the deterministic model worksheet also includes into three parts: (1) Zone 1 contains the input information required for geotechnical deterministic analyses, such as calculating the factor of safety, FS, of geotechnical structures; (2) Zone 2 allows geotechnical practitioners to develop calculation models for deterministic analyses of the geotechnical structure concerned in design; (3) Zone 3 calculates the system responses (e.g., $Y$ for SS and GSS) concerned in MCS-based full probabilistic design and provides other responses (e.g., FS) of interest as output. From an I-O perspective, the deterministic analysis worksheet takes a given set of uncertain parameters and possible designs as input and returns system responses (e.g., $Y$ or $F S$ ) as output. No probability concept is involved in the deterministic model worksheet, and it can be developed by geotechnical practitioners without background of probability and statistics. 


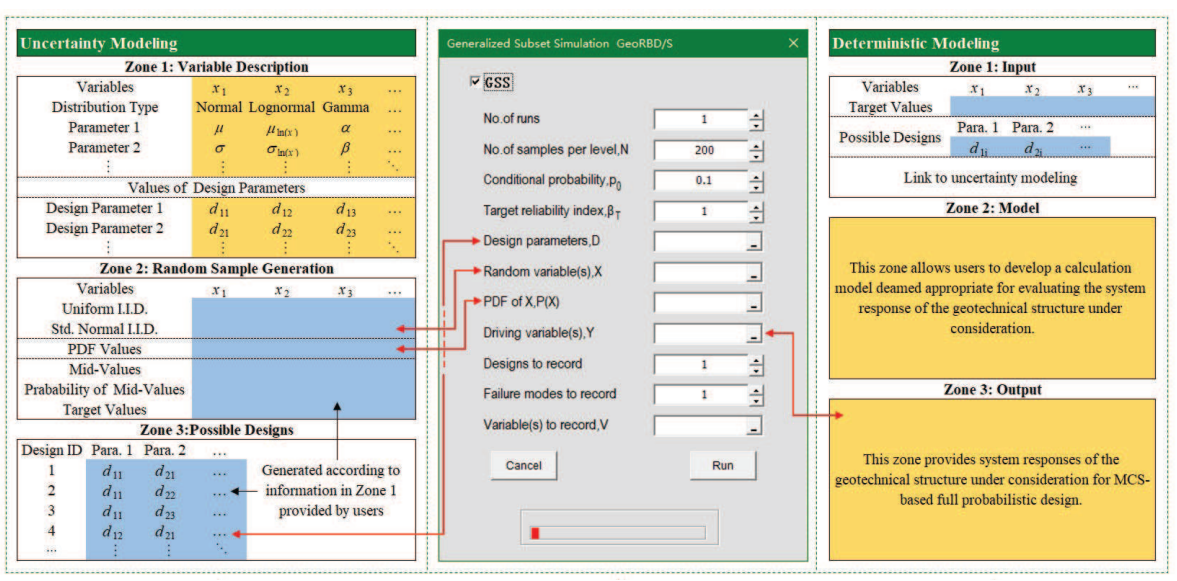

(a)Uncertainty model worksheet (b) MCS add-In for performing GSS (c) Deterministic model worksheet

When deterministic and uncertainty model worksheets are completed and linked together, MCS add-In is invoked to implement direct MCS, SS or GSS for full probabilistic design. For example, Figure 1(b) shows the GUI of the add-In for implementing GSS. After MCS add-In is set up, it invokes the uncertainty model worksheet and deterministic model worksheet to generate random samples and perform design calculations for each random sample, which are used to calculate $P\left(F \mid D^{(j)}\right)$ values of possible designs. Feasible designs are then determined as those with $P\left(F \mid D^{(j)}\right)<P_{T}$. Using GeoRBD/S, the uncertainty modeling and propagation are deliberately decoupled from the conventional deterministic analysis so that they can proceed as an extension of the deterministic analysis. This allows the deterministic and uncertainty model worksheets to be developed separately by personnel with different expertise and in a parallel manner, which are linked together and then processed by MCS add-In for the design. This permits geotechnical practitioners to instruct the spreadsheet to perform MCS-based full probabilistic design of geotechnical structures conveniently, reducing conceptual and mathematical complexity resulted from reliability algorithms.

\section{$5 \quad$ Illustrative Example}

This section illustrates the MCS-based full probabilistic design using a semi-gravity retaining wall example (Low 2005). As shown in Figure 2, the semi-gravity retaining wall example has a top width of $a$ and a bottom heel width of $b_{h}$ (or equivalently a bottom width $b=a+b_{h}$ ), and it has a height, $H$, of $6 \mathrm{~m}$. The back of the wall is vertical and the ground surface of the retained soil is inclined upwards at an angle, $\alpha$, of $10^{\circ}$. The retained soil has an internal friction angle, $\varphi$, and unit weight, $\gamma$, of $18 \mathrm{kN} / \mathrm{m}^{3}$. The wall is made of concrete with a density, $\gamma_{\text {wall }}$, of $24 \mathrm{kN} / \mathrm{m}^{3}$, and $\delta$ represents the interface friction angle between retained soil and the back of the wall. The wall sits on in-situ stiff clay providing sufficient bearing capacity so that the bearing capacity failure is not of concern (Low, 2005). The base resistance to sliding of the wall is attributed to the adhesion, $c_{a}$, between the wall base and the stiff clay. The $\varphi, \delta$, and $c_{a}$ are normally distributed. Their mean values are $35^{\circ}, 20^{\circ}$, and $100 \mathrm{kPa}$, respectively; and their COV values are $0.1,0.1$, and 0.15 , respectively. The $\varphi$ and $\delta$ are positively correlated with a correlation coefficient $\rho_{\varphi, \delta}$ of 0.8 .

Based on the above statistical information of $\varphi, \delta$, and $c_{a}$, direct MCS, SS, and GSS are applied to designing the retaining wall example, as presented in Section 2. The possible value of $a$ is considered varying from 0.2 to $0.6 \mathrm{~m}$ at an interval of $0.1 \mathrm{~m}$, and five possible values (i.e., $1.0,1.2,1.4,1.6$, and $1.8 \mathrm{~m}$ ) of $b_{h}$ are adopted, resulting in a total of 25 possible designs. When using direct MCS, a number of 25,000,000 samples were generated for expanded RBD. For SS and GSS, a number of 50000 and 5000 samples were generated in each simulation level, respectively, and the conditional probability $p_{0}$ is taken as 0.1 . For each random sample, the factors of safety of sliding and overturning modes of $D^{(j)}$ specified by $a$ and $b_{h}$ are calculated using equations given by Low (2005). The random samples simulated by the direct MCS, SS, and GSS and their corresponding factors of safety are then used to calculate the system failure probability $P_{f}^{(j)}$, sliding failure probability $P_{f, S}^{(j)}$, and overturning failure probability $P_{f, 0}^{(j)}$ of different possible designs. As shown in Figure 3, the results obtained from the three methods generally agree well with each other. Consider, for example, a system target failure probability $P_{T}=$ $1.35^{-3}$, as shown by the dot-dashed line in Figure 3(a). Feasible designs are those with $P_{f}{ }^{(j)}$ values below the 
dot-dashed line. In comparison with direct MCS-based expanded RBD, SS and GSS-based full probabilistic design require much less samples, leading to significant improvement of computational efficiency.

Results shown in Figure 3 were obtained under the original design scenario, where $\rho_{\varphi, \delta}=0.8$ and $c_{a}$ is normally distributed. Consider, for example, an updated design scenario, where $\rho_{\varphi, \delta}=0.5$ and $c_{a}$ is a lognormal random variable with a mean value of $100 \mathrm{kPa}$ and $\mathrm{COV}$ of 0.15 . Eq. (6) is used to obtain the updated failure probabilities of each possible design based on the direct MCS samples generated under the original design scenario so as to avoid re-running direct MCS for expanded RBD. Figure 4 shows the updated failure probabilities of different designs obtained from Eq. (6) by solid lines with open squares. For validation, direct MCS-based expanded RBD was also performed to re-evaluate the failure probabilities of the 25 possible designs under the updated design scenario, where a new set of 25,000,000 samples are generated for expanded RBD. Figure 4 also includes the results obtained from re-running the direct MCS-based expanded RBD by dashed lines with solid squares. It is shown that the updated failure probabilities and feasible designs obtained from the sample re-weighting technique (i.e., Eq. (6)) and re-running the direct MCS-based expanded RBD are in good agreement in this example. Using Eq. (6) allows determining feasible designs under different design scenarios based on direct MCS in a cost-effective manner, achieving efficient RBD updating using direct MCS.

For a given design, the random samples also provide information on reliability sensitivity and design points of different failure modes of the design. Figures 5(a) and (b) show the absolute values of $Z_{H}$ of $\varphi$ and $\delta$, and $c_{a}$ for the overturning and sliding failure modes of the design with $a=0.4 \mathrm{~m}$ and $b_{h}=1.4 \mathrm{~m}$. As shown in Figures 5(a) and (b), the $c_{a}$ has the smallest and largest absolute values of $Z_{H}$ for overturning and sliding failure modes, respectively. This indicates that the uncertainty in $c_{a}$ has the minimal effect on the overturning failure probability of the design with $a=0.4 \mathrm{~m}$ and $b_{h}=1.4 \mathrm{~m}$ while it affects significantly the sliding failure probability of the design. This is further confirmed by the results (see Figure 5(c)) obtained from Bayesian analysis described in Subsection 3.2. As shown in Figure 5(c), the conditional failure probability of the overturning failure mode of the design with $a=0.4 \mathrm{~m}$ and $b_{h}=1.4 \mathrm{~m}$ varies slightly as the $c_{a}$ increases. In contrast, the conditional failure probability of the sliding failure mode of the design reduces significantly as the $c_{a}$ increases. Such observations are consistent with those reported by Low (2005) based on FORM and design points of uncertain parameters for the same design.

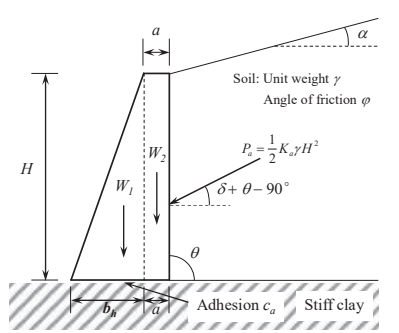

Figure 2. A semi-gravity retaining wall design example (After Low 2005)

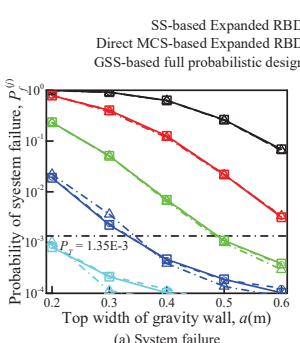

(a) System failure

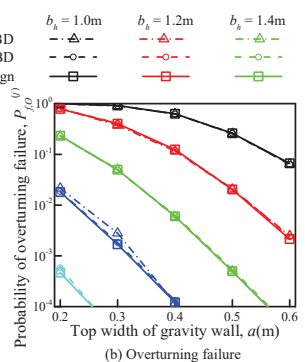

(b) Overturning failure

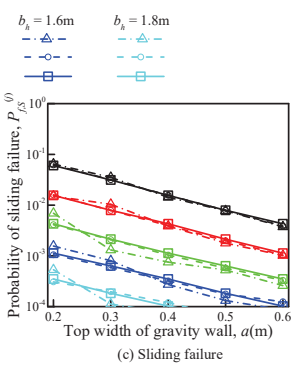

(c) Sliding failure

Figure 3. Failure probability of possible designs of the design example obtained from direct MCS, SS, and GSS

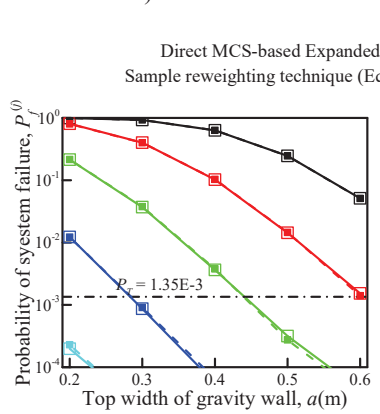

(a) System failure
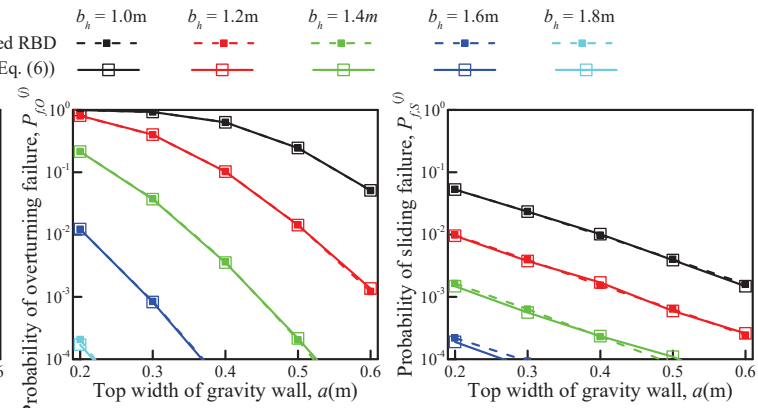

(b) Overturning failure

(c) Sliding failure

Figure 4. Updated failure probability of possible designs of the design example under the updated design scenario 
Table 1. Design points of overturning and sliding failure modes of the design with $a=0.4 \mathrm{~m}$ and $b_{h}=1.4 \mathrm{~m}$.

\begin{tabular}{|c|c|c|c|c|c|c|c|}
\hline \multirow[b]{2}{*}{ Failure mode } & \multirow{2}{*}{$\begin{array}{c}\text { Uncertain } \\
\text { Parameter } X_{k}\end{array}$} & \multicolumn{3}{|c|}{ Design point $X_{k}^{*}$} & \multicolumn{3}{|c|}{ Sensitivity parameter $n_{X k}$} \\
\hline & & $\begin{array}{c}\text { FORM } \\
\text { Low }(2005)\end{array}$ & Direct MCS & GSS & $\begin{array}{c}\text { FORM } \\
\text { Low }(2005)\end{array}$ & Direct MCS & GSS \\
\hline \multirow{3}{*}{$\begin{array}{l}\text { Overturning } \\
\text { failure }\end{array}$} & $\varphi^{*}\left({ }^{\circ}\right)$ & 26.4 & 26.3 & 26.5 & -2.449 & -2.476 & -2.433 \\
\hline & $\delta^{*}\left({ }^{\circ}\right)$ & 15.5 & 15.6 & 15.4 & -2.233 & -2.185 & -2.277 \\
\hline & $c_{\mathrm{a}}{ }^{*}(\mathrm{kPa})$ & 100 & 102.5 & 98.9 & 0.000 & 0.167 & -0.074 \\
\hline & $\varphi^{*}\left({ }^{\circ}\right)$ & 29.1 & 29.0 & 29.4 & -1.675 & -1.701 & -1.591 \\
\hline \multirow[t]{2}{*}{ Sliding failure } & $\delta^{*}\left({ }^{\circ}\right)$ & 17.2 & 17.2 & 16.9 & -1.397 & -1.417 & -1.577 \\
\hline & $c_{\mathrm{a}}{ }^{*}(\mathrm{kPa})$ & 60.9 & 61.0 & 60.1 & -2.609 & -2.599 & -2.660 \\
\hline
\end{tabular}

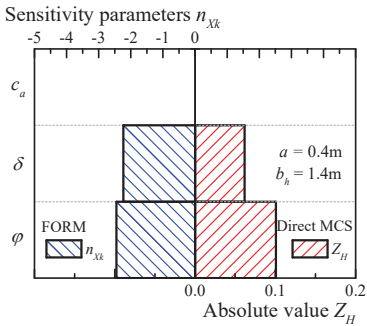

(a) Overturning failure mode

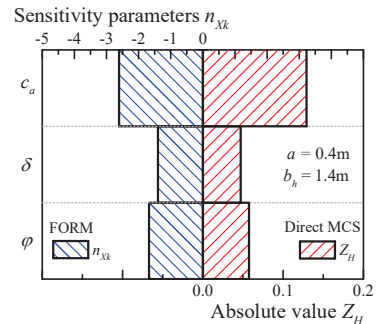

(b) Sliding failure mode

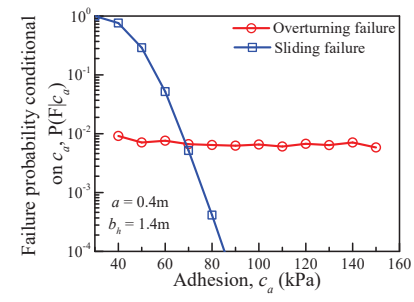

(c) Failure probability conditional on different $c_{a}$ values

Figure 5. Reliability sensitivity analysis results of uncertain parameters for the two failure modes of the design with $a=0.4 \mathrm{~m}$ and $b_{h}=1.4 \mathrm{~m}$ under the original design scenario.

Table 1 compares the design points (i.e., $\left.\left\{\varphi^{*}, \delta^{*}, c_{\mathrm{a}}^{*}\right\}\right)$ of overturning and sliding failure modes of the design with $a=0.4 \mathrm{~m}$ and $b_{h}=1.4 \mathrm{~m}$ identified from failure samples that are generated by direct MCS and GSS under the original design scenario with those obtained from FORM as reported by Low (2005). As shown in Table 1, design points identified from failure samples generated by direct MCS and GSS for the design are close to those obtained from FORM. The design points also contain the reliability sensitivity information of uncertain parameters. Based on the design point, the sensitivity parameter $n_{X k}$ of $X_{k}$ (i.e., $\left\{\varphi, \delta, c_{\mathrm{a}}\right\}$ ) is calculated as $\left(X_{k}^{*}-\mu_{k}\right) / \sigma_{k}$, where $X_{k}^{*}$ denotes the $X_{k}$ value of the design point. Table 1 shows the $n_{X k}$ values of $\varphi, \delta$, and $c_{\mathrm{a}}$ obtained from direct MCS, GSS, and FORM for overturning and sliding failure modes of the design with $a=$ $0.4 \mathrm{~m}$ and $b_{h}=1.4 \mathrm{~m}$. The $n_{X k}$ values are generally negative because the design point is located at the lower tail of the PDF of uncertain parameters except $c_{a}{ }^{*}$ for the overturning failure mode. For a given failure mode, the effect of $X_{k}$ on the failure probability becomes more significant as the value of $n_{X k}$ decreases. Figures 5(a) and (b) compare the values of $n_{X k}$ from FORM and the absolute values of $Z_{H}$ from direct MCS samples for overturning and sliding failure modes, respectively. It is shown that the value of $n_{X k}$ and the absolute value of $Z_{H}$ indicate the same order of reliability sensitivity of uncertain parameters. For the overturning failure mode, the reliability sensitivity decreases in an order of $\varphi, \delta, c_{\mathrm{a}}$ with the increase of $n_{X k}$ and the decrease of the absolute value of $Z_{H}$. In contrast, the reliability sensitivity decreases in an order of $c_{\mathrm{a}}, \varphi, \delta$ for the sliding failure mode.

\section{Concluding Remarks}

Monte Carlo simulation (MCS) provides a flexible and robust vehicle for geotechnical full probabilistic RBD. It allows representing complex probability distributions numerically using random samples and then performing complicated probabilistic reasoning in a convenient and discrete manner, in which each random sample is taken as the input of deterministic analysis models involved in design calculations. More importantly, this decouples uncertainty modeling and propagation from deterministic modeling so as to remove hurdles of reliability algorithms, rendering the extension of deterministic design analysis to MCS-based full probabilistic RBD a relatively trivial task, i.e., repeatedly calculating deterministic design models and counting the number of failure samples for different designs. Depending on MCS techniques (e.g., direct MCS, SS, and GSS) adopted in full probabilistic RBD, reliability calculations are performed in different ways, which have been implemented in an EXCEL add-In named GeoRBD/S to facilitate the design practice. Moreover, MCS samples contain more values beyond reliability calculations. Post-processing of MCS samples allows achieving efficient RBD updating under 
different design scenarios, obtaining reliability sensitivity information of uncertain parameters, and determining design points from failure samples that bridges full and semi- probabilistic RBD methodologies.

Last but not least, the number of random samples plays a vital role in MCS-based full probabilistic design, particularly the number of failure samples that are of interest in reliability calculations. Although SS and GSS are capable of efficiently generating a large number of failure samples, a potential panacea is emerging with fast advances of computing technologies, e.g., high performance computing. This makes it possible to incorporate more detailed uncertainty and deterministic modeling into MCS-based probabilistic analysis and RBD of geotechnical engineering systems and to explore rare events (e.g., failure events of feasible designs with high reliability) with relative ease even though direct MCS is used.

\section{Acknowledgments}

This work was supported by the National Key R\&D Program of China (Project No. 2016YFC0800200), the National Natural Science Foundation of China (Project Nos. 51879205, 51779189, and 51579190), and Young Elite Scientists Sponsorship Program by CAST (Project No. 2017QNRC001). The financial support is gratefully acknowledged. The authors also deeply appreciate many inspiring discussions on this topic with Professors Ivan Siu-Kui Au, Hong-Shuang Li and Kok Kwang Phoon.

\section{References}

Ang, A.H.-S. and Tang, W.H. (2007). Probability Concepts in Engineering: Emphasis on Applications to Civil and Environmental Engineering, John Wiley \& Sons, New York.

Au, S.K. and Wang, Y. (2014). Engineering Risk Assessment with Subset Simulation, John Wiley \& Sons, Singapore. ISBN: 978-1118398043.

Au, S.K., Cao, Z.J., and Wang, Y. (2010). Implementing advanced Monte Carlo simulation under spreadsheet environment. Structural Safety, 32(5), 281-292.

Au, S.K. and Beck, J.L. (2001). Estimation of small failure probabilities in high dimensions by subset simulation. Probabilistic Engineering Mechanics, 16(4), 263-277.

Baecher, G.B. and Christian, J.T. (2003). Reliability and Statistics in Geotechnical Engineering, John Wiley \& Sons, Chichester.

Cao, Z.J., Peng, X., Li, D.Q., and Tang, X.S. (2019). Full probabilistic geotechnical design under various design scenarios using direct Monte Carlo simulation and sample reweighting. Engineering Geology, 248, 207-219.

Ching, J. and Phoon, K.K. (2012). Value of geotechnical site investigation in reliability-based design. Advances in Structural Engineering, 15(11), 1935-1945.

Der Kiureghian, A., and Dakessian, T. (1998). Multiple design points in first and second-order reliability. Structural Safety. 20(1), 37-49.

Gao, G.H., Li, D.Q., Cao, Z.J., Wang, Y., and Zhang, L. (2019). Full probabilistic design of earth retaining structures using generalized subset simulation. Computers and Geotechnics, 112, 159-172.

ISO2394:2015. General Principles on Reliability for Structures, Geneva, International Organization for Standardization.

Li, D.Q., Shao, K.B., Cao, Z.J., Tang, X.S., and Phoon, K.K. (2016). A generalized surrogate response aided-Subset Simulation approach for efficient geotechnical reliability-based design. Computers and Geotechnics, 74, 88-101.

Li, D.Q., Zhang, F.P., Cao, Z.J., Zhou, W., Phoon, K.K., and Zhou, C.B. (2015). Efficient reliability updating of slope stability by reweighting failure samples generated by Monte Carlo simulation. Computers and Geotechnics, 69, 588-600.

Li, H.S., Ma, Y.Z., and Cao, Z.J. (2015). A generalized Subset simulation approach for estimating small failure probabilities of multiple stochastic responses, Computers \& Structures, 153, 239-251.

Low, B.K. and Phoon, K.K. (2015). Reliability-based design and its complementary role to Eurocode 7 design approach. Computers and Geotechnics, 65, 30-44.

Low, B.K. (2005). Reliability-based design applied to retaining walls. Géotechnique, 55(1), 63-75.

Low, B.K. and Tang, W.H. (1997). Efficient reliability evaluation using spreadsheet. Journal of Engineering Mechanics, 123(7), 749-752

Phoon, K.K., Retief, J.V., Ching, J., Dithinde, M., Schweckendiek, T., Wang, Y., and Zhang, L.M. (2016). Some observations on ISO2394:2015 Annex D (Reliability of Geotechnical Structures). Structural Safety, 62, 24-33.

Rackwitz, R. and Fiessler, B. (1978). Structural reliability under combined random load sequences. Computers and Structures, 9, 484-94.

Shinozuka, M. (1983). Basic analysis of structural safety. Journal of Structural Engineering, 109(3), 721-740.

Wang, Y. and Cao, Z.J. (2015). Practical reliability analysis and design by Monte Carlo Simulation in spreadsheet. Risk and Reliability in Geotechnical Engineering, Phoon, K.K., Ching, J.Y. eds., Taylor \& Francis CRC Press, 301-335.

Wang, Y. and Cao, Z.J. (2013). Expanded reliability-based design of piles in spatially variable soil using efficient Monte Carlo simulations. Solis and Foundations, 53(6), 820-834.

Wang, Y. (2011). Reliability-based design of spread foundations by Monte Carlo Simulations. Géotechnique, 61(8), 677-685.

Wang, Y., Au, S.K., and Kulhawy, F.H. (2011). Expanded reliability-based design approach for drilled shafts, ASCE Journal of Geotechnical and Geoenvironmental Engineering, 137 (2), 140-149.

Wang, Y., Cao, Z., and Au, S.K. (2010). Efficient Monte Carlo simulation of parameter sensitivity in probabilistic slope stability analysis. Computers and Geotechnics, 37(7-8), 1015-1022. 